\title{
Accumulation and Control of Spin Waves in Magnonic Dielectric Microresonators by a Comb of Ultrashort Laser Pulses
}

\section{A. E. Khramova ( $\sim$ ae.khramova@gmail.com )}

Russian Quantum Center

M. Kobecki

TU Dortmund

I. A. Akimov

TU Dortmund

I. V. Savochkin

Russian Quantum Center

M. A. Kozhaev

Russian Quantum Center

\section{A. N. Shaposhnikov}

Vernadsky Crimean Federal University

V. N. Berzhansky

Vernadsky Crimean Federal University

\section{A. K. Zvezdin}

Prokhorov General Physics Institute RAS

\section{Bayer}

TU Dortmund

\section{I. Belotelov}

Russian Quantum Center

\section{Research Article}

Keywords: Spin waves, magnetic microresonators, magnonics, Ultrashort Laser Pulses

Posted Date: June 23rd, 2021

DOl: https://doi.org/10.21203/rs.3.rs-637840/v1

License: (c) (i) This work is licensed under a Creative Commons Attribution 4.0 International License.

Read Full License 


\title{
Accumulation and control of spin waves in magnonic dielectric microresonators by a comb of ultrashort laser pulses
}

\author{
A.E. Khramova ${ }^{1,2^{*}}$, M. Kobecki ${ }^{3}$, I.A. Akimov ${ }^{3,6}$, I.V. Savochkin ${ }^{1,2}$, M.A. Kozhaev ${ }^{1,4}$, A.N. \\ Shaposhnikov $^{5}$, V.N. Berzhansky ${ }^{5}$, A.K. Zvezdin ${ }^{1,4,7}$, M.Bayer ${ }^{3,6}$, and V.I. Belotelov ${ }^{1,2}$ \\ ${ }^{1}$ Russian Quantum Center, 45, Skolkovskoye shosse, Moscow, 121353, Russia \\ ${ }^{2}$ Faculty of Physics, Lomonosov Moscow State University, Leninskie Gory, Moscow 119991, Russia \\ ${ }^{3}$ TU Dortmund, Experimentelle Physik 2, D-44221 Dortmund, Germany \\ ${ }^{4}$ Prokhorov General Physics Institute RAS, 38 Vavilov Street, Moscow 119991, Russia \\ ${ }^{5}$ Vernadsky Crimean Federal University, 4 Vernadskogo Prospekt, Simferopol, 295007, Russia \\ ${ }^{6}$ Ioffe Institute Russian Academy of Sciences, 194021 St. Petersburg, Russia \\ ${ }^{7} \mathrm{NTI}$ Center for Quantum Communications, National University of Science and Technology MISiS, Leninsky \\ Prospekt 4, Moscow, 119049, Russia \\ *ae.khramova@gmail.com
}

\begin{abstract}
Spin waves in magnetic microresonators are at the core of modern magnonics. Here we demonstrate a new method of tunable excitation of different spin wave modes in magnetic microdisks by using a train of laser pulses coming at a repetition rate higher than the decay rate of spin precession. The microdisks are etched in a transparent bismuth iron garnet film and the light pulses influence the spins nonthermally through the inverse Faraday effect. The high repetition rate of the laser stimulus of $10 \mathrm{GHz}$ establishes an interplay between the spin wave resonances in the frequency and momentum domains. As a result, the excitation efficiency of different spin modes can be tuned by a small variation of the external magnetic field. An additional degree of freedom is provided by scanning the laser spot within the microdisk area. This makes the proposed method for spin wave excitation advantageous for the forthcoming application of magnonics for telecommunication and quantum technologies.
\end{abstract}

\section{Introduction}

Currently, research in the field of magnonics is of great importance. The practical application of spin waves is being rapidly developed as an alternative to existing methods of transmitting digital information as well as for use in quantum communications $^{1,2}$. These days, the first experimental developments of magnon logic systems have already been obtained ${ }^{3,4}$. They are based, for example, on the interaction of spin waves with domain walls of various chiralities, which reduces energy dissipation ${ }^{5}$. The development of spin resonators and interferometers for telecommunication devices is also actively underway $^{6-8}$. For most of practical magnonics implementations, magnetic dielectrics play a key role as their Gilbert damping is much lower than for magnetic metals. Among them, yttrium iron garnet thin films demonstrate a record performance, ${ }^{9,10}$. For example, yttrium iron garnet spheres of millimeter size were recently coupled to superconducting qubits in a microwave cavity to provide control and quantum data storage ${ }^{11}$.

Usually, spin waves are excited by microwave antennas ${ }^{12-14}$. This method has strong constrictions in tunability. For example, to change the position of the region where the spin waves are excited in a sample, a mechanical movement is necessary which limits operation rates and introduces additional noise. Apart from that, microwave antennas influence on relatively large part of a magnetic sample which worsens localization of the spin wave source. In this respect, optical means involving femtosecond laser pulses hold a large promise as they allow one to solve most of these obstacles ${ }^{15-23}$. Ultrashort laser pulses disturb the equilibrium magnetic state of the sample either via non-thermal effects like the inverse Faraday and Cotton-Mouton effect, or by the effect of the photoinduced magnetic anisotropy, or through the thermal effect of ultrafast demagnetization ${ }^{24}$. The former are mostly prominent for magnetic dielectrics, for example, bismuth iron garnets ${ }^{25}$ as they possess low optical absorption. The inverse Faraday effect arises due to the stimulated Raman scattering of light by magnons which can be understood in terms of the circularly polarized light creating an effective magnetic field along the light wavevector. A focused laser beam influences only the spins within the laser spot and during the pulse propagation through the sample. However, it is 
sufficient to trigger the magnetization precession. On a picosecond time scale, adjacent spins get also involved through the magnetic dipole-dipole and exchange interactions and the magnetic oscillations propagate away from the illuminated area in the form of spin waves.

Among the drawbacks of the optical method are the relatively broad spectrum of the launched spin waves and their small amplitudes. Usually, laser pulses excite the spin dynamics in a single pulse regime, where the magnetization precession started by one pump pulse completely decays before the arrival of the next pulse. Excitation in the multiple pulse regime where the time interval between pulses is shorter than the decay time is much more advantageous. Recently, it was demonstrated that the periodic laser impact on spins provides a kind of accumulation of the spin waves whose spectrum and directionality can be modified by a slight variation of the applied magnetic field or the pulse repetition rate ${ }^{16}$. Moreover, in this case the spin waves are excited in a frequency range much narrower than for single pulse excitation and with amplitudes prominently enhanced. Indeed, a train of fs-laser pulses provides a kind of resonance in the frequency domain and thus singles out spin wave frequencies that are multiples to the pulse rate. Taking into account that self-mode-locked solid state lasers are capable of generating optical pulses with the repetition rates up to several tens of $\mathrm{GHz}$ such approach enables optical generation of high frequency sub-THz magnons.

On the other hand, magnetic structures like magnonic waveguides, microresonators and magnonic crystals have become crucial elements of modern magnonics ${ }^{26-34}$. Multiple spin wave reflections from the boundaries and interfaces lead to resonances in the momentum domain. As a result, complex interference patterns are formed and additional resonances in the spin wave spectra appear. Magnonic microstructures are necessary for expanding the capabilities of magnonic devices as the micro- and nanostructuring enrich magnonic spectra and open new doors for their engineering. Moreover, magnonic microspheres and microdisks play the role of cavities for both magnons and photons and therefore are currently used for quantum magnonics platforms ${ }^{11}$.

Here we apply the method of spin wave accumulation by periodic laser pulses to magnetic microdisks to investigate the interplay between spin wave resonances in the frequency and momentum domains. In particular, we study magnetostatic spin waves in iron-garnet microdisks of different diameters excited by a sequence of ultrashort laser pulses. The train of laser pulses provides a cumulative effect, leading to quasi-stationary magnonic oscillations in the microdisks, which allows us to observe interference effects and to excite different magnonic modes. This is not possible for conventional excitation in the single pulse regime where every next pulse hits the sample after full relaxation of the spin waves generated by the previous pulse. Sweeping the external magnetic field switches between different modes. The efficiency of the mode excitation depends on its frequency and becomes maximal, when the frequency is a multiple of the pulses repetition rate. Scanning the laser spot across the microdisk area also modifies the spin mode amplitudes, providing additional room for magnonic tunability.

\section{Results and discussion}

The multiple pulse impact on the magnetization in the microdisks initiates a quasi-stationary process of magnetization precession (Fig. 3(b)). The magnetic oscillations excited by a pump pulse don't noticeably decay before the consecutive pulse hits the samples. However, their amplitudes strongly depend on the magnetic field $H$ (Fig. 3(c)). Moreover, for some disks this dependence has a double peak structure (Fig. 3(c), red circles).

We start the detailed studies from the 300- $\mu \mathrm{m}$ microdisk and illuminate its central part by the laser spot of much smaller diameter. Therefore, the influence of the disk boundaries can be neglected and the disk can be considered approximately as an infinite smooth magnetic film. The observed spin dynamics is a result of the excited magnetostatic spin waves. This is confirmed when the position of the pump and probe beam spots are separation, i.e. the observation area is moved away from the illuminated one: the oscillations are still observed but their amplitude decays with the distance between pump and probe spots (Fig. 1(a), compare the amplitude versus magnetic field plots of different color). The signal is hardly visible $20 \mu \mathrm{m}$ apart from the excitation spot. Actually, the observed short-range propagation of the spin waves results mostly from the dephasing effects appearing due to the relatively broad spectrum of the spin waves launched by the tightly focused laser pulses.

The dependence of the precession phase on the distance between the pump and probe spots measured at different magnetic fields allows one to derive the dispersion of the spin wave wavelength on the magnetic field ${ }^{9}$ (Fig. 1(b)). Apart from that, the spin wave dispersion $\omega(k)$ can be extracted (Fig. 1(c)) which corresponds to BVMSW since their frequency decreases for larger wavenumbers.

Figure 1(a) also indicates that the amplitude of the magnetization precession depends strongly on the magnetic field and has a resonant shape. The resonant amplification of the precession amplitude appears due to the synchronization of the instant pump pulses driving the magnetization with the time interval $T$ and the magnetization precession at frequency $\omega^{16}$.

Circularly polarized light influences the magnetization in magnetic dielectrics mainly through the inverse Faraday effect which can be described in terms of an effective magnetic field $H_{F}$, acting on the spins during the pulse propagation through the sample $^{35}$. Since the pulse duration $\Delta \mathrm{t}$ is sufficiently small compared to the laser pulse repetition period $(\Delta t \ll T)$, the field of the inverse Faraday effect can be represented as a series of $\delta$-functions: $H_{F}(t)=h \Delta t \sum_{l=0}^{+\infty} \delta(t-l T)$, where $h$ is the field 
amplitude, and $l$ is an integer. Solving the Landau-Lifshitz-Gilbert equation written in spherical coordinates for the periodic process of period $T$ gives the spin precession in the form of $\theta(t)=\theta_{0} \sin (\omega t+\xi) e^{-t / \tau_{0}}$ in the interval $l T<t<(l+1) T$.Here $\omega=\omega_{0} \sqrt{1-\left(2 \pi v_{0} \tau_{0}\right)^{-1}} \approx \omega_{0}, \omega_{0}=\sqrt{\omega_{H}\left(\omega_{H}+\omega_{d}-\omega_{a}\right)}, \tau_{0}=2 / \alpha\left(2 \omega_{H}+\omega_{d}-\omega_{a}\right), \omega_{H}=\gamma H, \omega_{d}=\gamma H_{d}, \omega_{a}=2 \gamma K_{u} / M$, $\theta_{0}$ and $\xi$ are the amplitude and initial phase of the oscillations, respectively ${ }^{15}$. The precession amplitude is given by

$$
\theta_{0}=\gamma^{2} H h \Delta t / \omega\left(1-2 e^{-T / \tau_{0}} \cos \omega T+e^{-2 T / \tau_{0}}\right)^{-1 / 2}
$$

Therefore, the amplitude reaches maximum values at $\omega=2 \pi m / T=\omega_{r}$, where $m$ is an integer and the dependence $\theta(H)$ should have a resonance shape. Here we deal with pulses coming at a high repetition rate, so that $T / \tau_{0} \ll 1$ and the resonances are relatively narrow, i.e. $\Delta \omega T \ll 1$, where $\Delta \omega$ is the resonance width. In this case, Eq.(1) in the vicinity of the resonance simplifies to a Lorentzian shape:

$$
\theta_{0}=\gamma^{2} H_{r o} h \Delta t /\left(2 \pi m\left(\left(\omega-\omega_{r}\right)^{2}+\tau_{0}\right)^{1 / 2}\right)
$$

where

$$
H_{r 0}=2 \pi m / \gamma T-\left(H_{d}-2 K_{u} / M\right) / 2
$$

is the external magnetic field of the resonance in the frequency domain. These expressions describe the observed spin dynamics in the limit of small wavenumbers $k$ of the spin waves $(k R \ll 1$, where $R$ is the size of the monitored area).

The magnetic field strength can be adjusted such that one gets only one precession cycle between two consecutive pulses $(m=1)$, then the damping of the precession is hardly visible. This is the case of our experiments (Fig. 3(b)). Tuning the external magnetic field varies the precession frequency, allowing one to finely synchronize it with the laser pulse train repetition rate. The highest amplitude of the precession should be achieved when its period coincides with the time interval between the pulses, i.e. at $H_{r 1}=2.96 \mathrm{kOe}$. This corresponds well with the position of the maximum in the experimental dependence of $\theta_{0}(H)$ in Fig. 1(a), blue circles, at $2.98 \mathrm{kOe}$.

Some deviations and the nonsymmetric shape of the resonance $\theta_{0}(H)$ ) are due to the excitation of the BVMSW with non-negligible $k$. Actually, the focused laser beam of radius $r$ excites BVMSW with wavevectors $k<2 / r$. Due to the BVMSW dispersion $\omega(k)$ the spectrum of the excited spin waves is broadened: $\omega(1 / r)<\omega<\omega_{0}$.

Therefore, the resonance conditions for the different spin wave harmonics occur at slightly different magnetic fields broadening the resonance of $\left.\theta_{0}(H)\right)$ and shifting it slightly.

The influence of the microdisk boundaries can be studied for the $300-\mu \mathrm{m}$ structure as the spin wave dispersion in the disk is shown (Fig. 1(b)). Let us compare four positions of the pump and probe spot in the disk (Fig. 1(d)): (I) at the center of the disk (red curve); (II) at a distance $L=15$ - $\mu$ m apart from the edge of the disk along the direction of the magnetic field, i.e. along the $x$ axis (black curve); (III) at a distance $L=7 \mu \mathrm{m}$ apart from the edge of the disk along the $\mathrm{x}$ axis (green curve), and (IV) at a distance $L=7 \mu \mathrm{m}$ apart from the edge of the disk in a direction perpendicular to the magnetic field, i.e. along the $y$ axis.

For the illumination at the microdisk center the amplitude dependence $\theta_{0}(H)$ has a single peak at $H_{r 1}=2.98 \mathrm{kOe}$, while for the positions (II) and (III) $\theta_{0}(H)$ has two peaks. The most pronounced one at around $2.98 \mathrm{kOe}$ is due to the synchronization of the magnetization oscillations and pump pulses, as we discussed earlier.

However, the most important feature of the $\theta_{0}(H)$ dependence is the second peak (see Fig. 1(d), black and green dots). Its position shifts to higher fields for the light spots shifting closer to the microdisk edge. For the $L=15 \mu \mathrm{m}$ separation from the edges (point (II)) the peak appears at the magnetic field of $3.06 \mathrm{kOe}$, while for $L=7 \mu \mathrm{m}$ (point (III)) at $3.14 \mathrm{kOe}$. Apart from that, for the point (III) there is also a feature at $3.04 \mathrm{kOe}$, that broadens and shifts the main resonance to higher magnetic fields the main resonance. At the same time, shift of the pump-probe beams toward the disk boundaries in the direction perpendicular to $H$ (point (IV)) doesn't give rise to any additional peaks (Fig. 1(d), blue dots).

The observed behavior of the spin wave amplitude hints at interference between spin waves directly excited by the pump pulses and reflected from the microdisk edges. In detail, the spin waves excited at the center of the 300- $\mu \mathrm{m}$ disk don't reach the disk boundaries since their propagation length is much smaller, of the order of a few tens of microns. As a result, no interference is possible. However, if the spin waves are launched a few microns apart from the microdisk edge, namely at a distance $L$, then the interference becomes possible and should be most efficient for the spin wave wavelengths $\lambda \sim 2 L / n$ for $n$ is an integer, so that the phase difference between the spin waves directly excited by the pump and the one reflected from the edges is close to 0 or $2 \pi$.

At $H_{r 2}=3.06 \mathrm{kOe}$ (the resonance field at point (II)) the average wavelength of the BWMSW is $=15 \mu \mathrm{m}$, while at $H_{r 2}=3.14$ kOe (the resonance field at point (III)) $\lambda=7 \mu \mathrm{m}$ (Fig. 1(b)). In both cases it nicely agrees with the condition $L \sim \lambda n / 2$ for $n=2$. Consequently, the additional peaks are indeed a result of spin wave interference. The absence of the second peak for the shift along the $y$-axis (point (IV)) is then explained by the character of the BVMSW which propagate mostly along the magnetic 


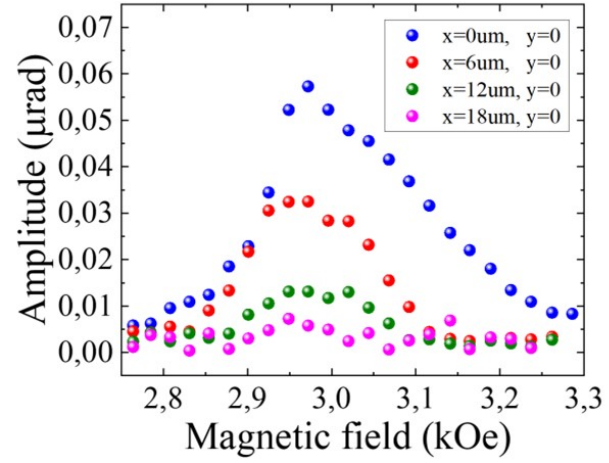

(a)

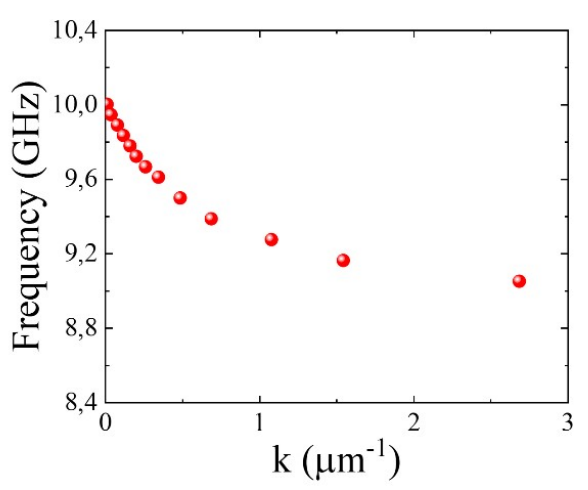

(c)

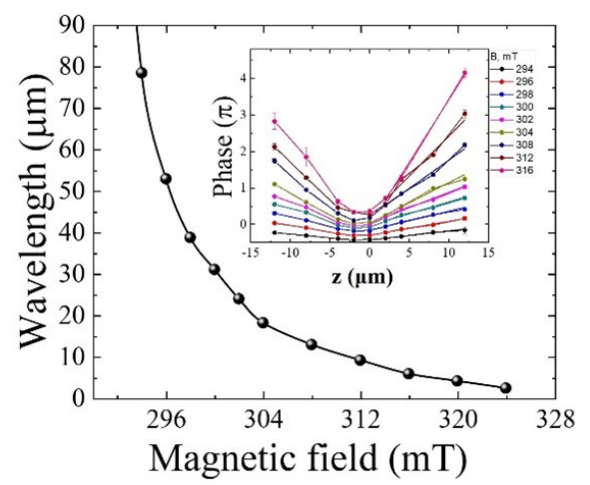

(b)

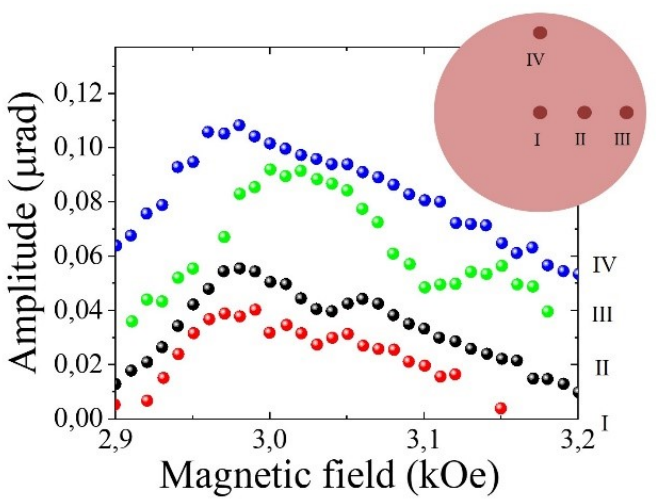

(d)

Figure 1. Spin waves excited in the 300- $\mu \mathrm{m}$ disk. (a) Dependence of the spin waves amplitude on the external magnetic field for different positions of the probe beam relative to the pump one. The pump beam is located in the center of the disk. (b) Dependence of the spin waves wavelength on the external magnetic field derived from the behavior of the spin wave phase along the $\mathrm{x}$-axis (see inset) measured for different magnetic fields. (c) Experimentally evaluated spin wave dispersion corresponding to backward volume magnetostatic spin waves (BVMSW). (d) Dependence of the spin waves amplitude on the applied magnetic field at four different positions of the pump and probe beams. The inset shows these four positions on the microdisk. The pump fluence is $5,2 \mu \mathrm{J} / \mathrm{cm}^{2}$.

field, i.e. along the x-axis. This makes the reflection from the boundary near point IV negligibly small and prevents any notable interference .

Apart from the resonance for $n=2$ there should also be the resonance for $n=1$. In accordance with Fig. 1(b) at the point (II) this resonance is expected for $H_{r 1}=3.00 \mathrm{kOe}$. This field is very close to $H_{r 0}=2.98 \mathrm{kOe}$ and for that reason the resonance is not so pronounced.

At the point (III) which is twice closer to the microdisk edge the second order resonance $(n=2)$ is shifted to $H_{r 2}=3.14 \mathrm{kOe}$, since it is the magnetic field providing spin waves of wavelength $\lambda=7 \mu \mathrm{m}$. The first order resonance $(n=1)$ is expected at $H_{r 1}=$ $3.06 \mathrm{kOe}$. This explains the feature on the green curve around $3.05 \mathrm{kOe}$ which broadens and shifts the main resonance.

As the size of the disks decreases, additional maxima of the precession amplitude appear as well whose origin can be associated with interference. However, in this case, the size of the disk is comparable to the propagation length of the spin wave, and the phenomenon of interference becomes more complicated. The strong influence of the boundaries is manifested in the behavior of the magnetization oscillation amplitude for different disks (Fig. 2(a)). For smaller disks the field $H_{r 0}$ starts to increase. For example, for the $20-\mu \mathrm{m}$ disk $H_{r 0}=3.01 \mathrm{kOe}$ (Fig. 2(a), red circles). The peak shift by 30 Oe is due to the smaller average demagnetizing field $H_{d}$ of the microdisk with respect to the film. Eq.(3) gives the correct value of $H_{r 0}$ if the average demagnetizing field of the $20-\mu \mathrm{m}$ disk is $H_{d}=1.24 \mathrm{kOe}$.

Notably, for the $20-\mu \mathrm{m}$ disk a second peak appears at $H_{r} 2=3.13 \mathrm{kOe}$. Its origin can be understood from the spatial distribution of $\theta_{0}(x, y)$, calculated by the micromagnetic modeling program Mu-Max with the cell dimensions of $0.25 \times 0.25 \times$ $1.1 \mu^{3}$ (Fig. 2(b-e)). Periodic pumping at $10 \mathrm{GHz}$ repetition rate singles out different magnetostatic modes of the disk formed 


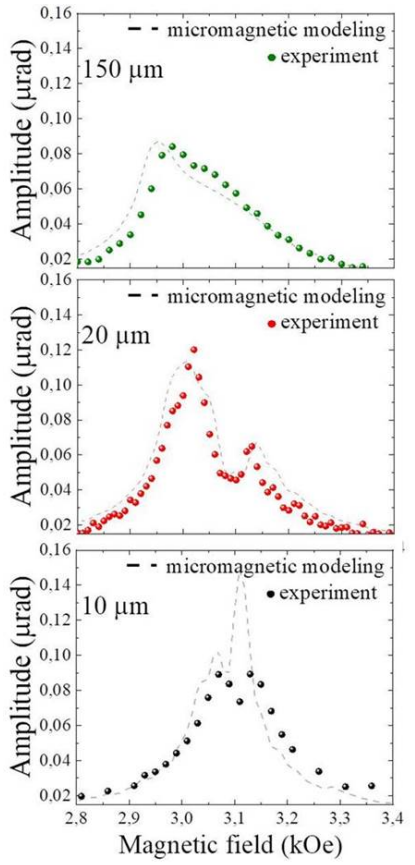

a

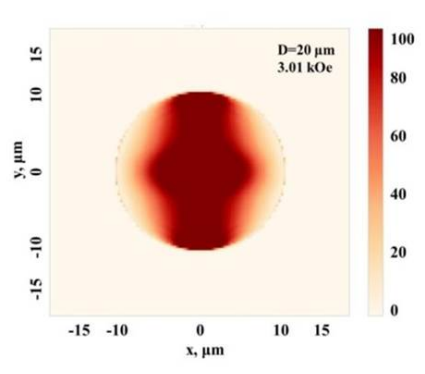

b

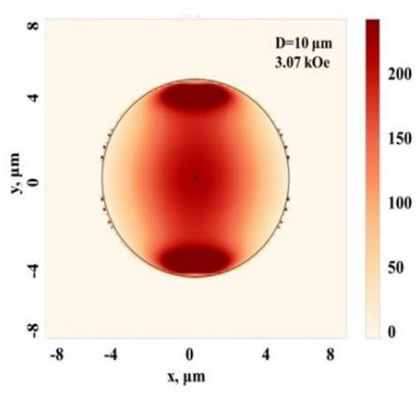

d

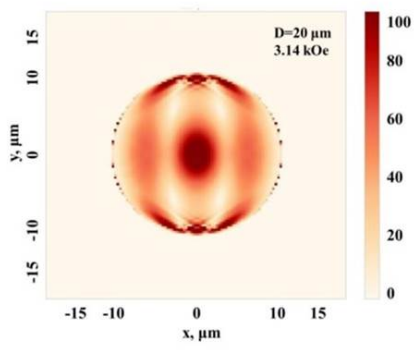

c

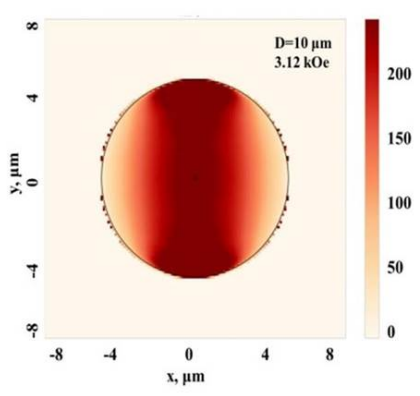

e

Figure 2. Fig 3. (a) Experimental data of the amplitude of the magnetization precession dependence on the magnetic field for disks of 150, 20 and $10 \mu \mathrm{m}$ diameter (circles), as well as micromagnet modeling results of the amplitude of the magnetization precession (dashed lines), the energy density is $5.2 \mu \mathrm{J} / \mathrm{cm}^{2}$. (b-e) Spatial distribution of the spin wave amplitude for the $20 \mathrm{~m}$ disk at $\mathrm{H}=3.01 \mathrm{kOe}(\mathrm{b})$ (the lower field resonance peak), and at $\mathrm{H}=3.14 \mathrm{kOe}$ (c) (the higher field resonance peak), for the 10 $\mu \mathrm{m}$ disk at $\mathrm{H}=3.07 \mathrm{kOe}(\mathrm{d})$ (the lower field resonance peak), and at $\mathrm{H}=3.12 \mathrm{kOe}(\mathrm{e})$ (the higher field resonance peak).

by standing spin waves. As the modes are formed by BVMSWs, the interference takes place along the external magnetic field, i.e. one or several amplitude maxima appear in $\mathrm{x}$-axis direction. Thus, the mode excited at $H_{r 0}=3.01 \mathrm{kOe}$ is the standing spin wave of the first order $\left(n_{x}=1\right)$, while the mode at $H_{r 2}=3.13 \mathrm{kOe}$ is of the third order $\left(n_{x}=3\right)$.

The amplitude of the spin precession in the 10- $\mu \mathrm{m}$ disk also has two peaks at $3.07 \mathrm{kOe}$ and $3.12 \mathrm{kOe}$. At a magnetic field of $3.12 \mathrm{kOe}$ the first-order $\left(n_{x}=1\right)$ mode of the BMSW is excited (Fig. 2(e)), while origin of the lower field peak at $3.07 \mathrm{kOe}$ should be different since the amplitude distribution doesn't correspond to any BMSW modes and hints on the interference in y-axis direction (Fig. 2(d)). It corresponds to the other type of spin waves, namely magnetostatic surface spin waves (MSSW). The efficiency of MSSW excitation by laser pulses increases for smaller beam diameters ${ }^{36}$. In the current studies the pump beam diameter was kept the same for all microdisks. Nevertheless, the distribution of light intensity in the laser beam cross-section might be modified for the microdisks with a size comparable to the beam diameter. This is the case for the smallest disks in our studies which are $10 \mu \mathrm{m}$ in diameter as the pump beam's diameter is $7 \mu \mathrm{m}$. The microdisk boundaries distort the Gaussian beam profile reducing its effective diameter. The latter increases the range of the spin wavenumbers that are attainable by the laser beam which favors the excitation of MSSWs. Therefore, the lower field resonance peak is due to the first order $\left(n_{y}=1\right)$ MSSW mode.

\section{Conclusions}

To conclude, we have studied the excitation of spin wave modes by a sequence of ultrashort laser pulses impinging on the magnetic microdisks at the ultimately high repetition rate of $10 \mathrm{GHz}$. The focused laser spot acts as a localized stimulus for launching the spin oscillations which significantly sets it apart from conventional microwave generation means. The high repetition rate leads to spin wave accumulation. Due to the reflection of the spin waves from the disk boundaries the interference and standing mode formation take place. An additional degree of tunability might be achieved by scanning the laser spot across the microdisk area which provides different interference patterns. The experimental results were obtained with the laser focused to a spot of $7 \mu \mathrm{m}$ in diameter. If a tighter focusing is used, around $1 \mu \mathrm{m}$ in diameter, then it might be possible to scan across a smaller microdisk and launch not only odd modes, but even modes as well. This makes the proposed method for spin wave excitation advantageous for the forthcoming application of magnonics for telecommunication and quantum technologies. 


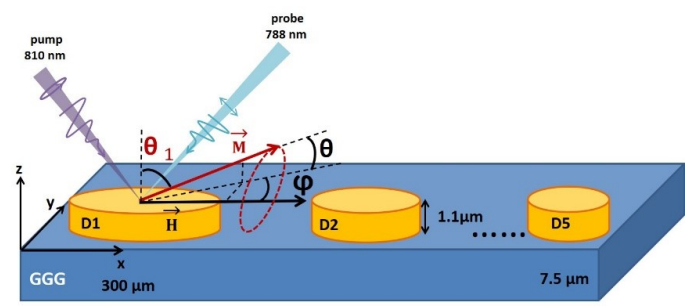

(a)

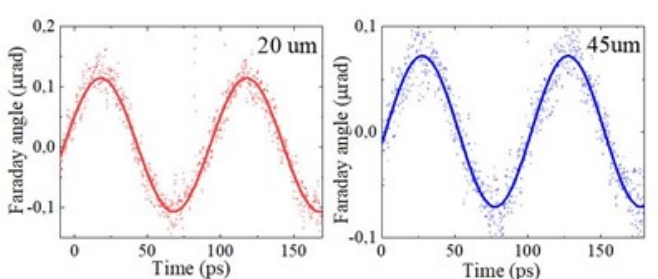

(b)

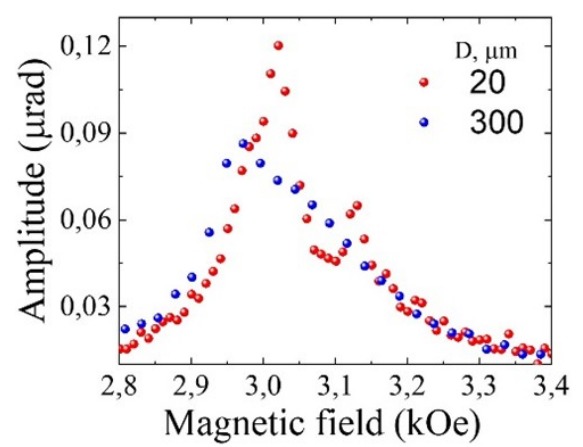

(c)

Figure 3. Spin waves excited in the 300- $\mu \mathrm{m}$ disk. (a) Dependence of the spin waves amplitude on the external magnetic field for different positions of the probe beam relative to the pump one. The pump beam is located in the center of the disk. (b) Dependence of the spin waves wavelength on the external magnetic field derived from the behavior of the spin wave phase along the X-axis (see inset) measured for different magnetic fields. (c) Experimentally evaluated spin wave dispersion corresponding to backward volume magnetostatic spin waves (BVMSW). (d) Dependence of the spin waves amplitude on the applied magnetic field at four different positions of the pump and probe beams. The inset shows these four positions on the microdisk. The pump fluence is $5,2 \mu \mathrm{J} / \mathrm{cm}^{2}$.

\section{Methods}

The experimental studies were conducted on a $1.1 \mu \mathrm{m}$-thick magnetic dielectric film of Bi-substituted rare-earth iron garnet $(\mathrm{BiLuGdEu})_{3} \mathrm{Fe}_{5} \mathrm{O}_{12}$, grown on a gadolinium gallium garnet substrate with the crystallographic orientation (111). The saturation magnetization is $4 \pi M_{s}=1300 \mathrm{G}$, the uniaxial anisotropy constant is $K_{u}=3 \cdot 10^{3} \mathrm{erg} / \mathrm{cm}^{3}$, and the Gilbert dissipation constant is $\alpha=4 \cdot 10^{-3}$. Microdisks of various diameters were etched in the film down to the substrate using a photolithography and wet etching process (Fig.3(a)).

To study the laser excitation of spin waves in the disks, the sample is placed in a saturating in-plane external magnetic field and a pump-probe method based on asynchronous optical sampling (ASOPS) (Supplementary, Fig. 4) is implemented ${ }^{37}$. The circularly polarized laser pump pulses of duration $50 \mathrm{fs}$ at the wavelength $\lambda_{\text {pump }}=810 \mathrm{~nm}$ are focused in a spot of $9 \mu \mathrm{m}$ in diameter and hit the sample each 100 ps, initiating the spin dynamics, which can be described in a spherical coordinate system by the polar and azimuth angles $\theta_{1}$ and $\phi$, respectively, where the z-axis is directed along the film normal (Fig. 3(a)). The spin precession takes place around the external magnetic field directed along the $\mathrm{x}$-axis. The spins remain close to the equilibrium direction along the $\mathrm{x}$-axis and follow an elliptical trajectory so that $\theta=\pi / 2-\theta_{1} \ll 1$ and $\phi \ll 1$. The spin precession is monitored via the Faraday effect by the probe beam at $\lambda_{\text {probe }}=780 \mathrm{~nm}$ wavelength focused in a spot of $7 \mu \mathrm{m}$ in diameter. The probe beam arrives at the sample at a variable time delay with respect to the pump which allows to observe the magnetization vector changes with temporal resolution. The rotation of the probe polarization by the Faraday angle $\Phi$ provides the angle $\theta$ : $\theta=\Phi / \Phi_{0}$, where $\Phi_{0}$ is the Faraday angle for the magnetic film fully saturated out-of-plane.

\section{References}

1. Gündoğan, M., Ledingham, P. M., Kutluer, K., Mazzera, M. \& De Riedmatten, H. Solid state spin-wave quantum memory for time-bin qubits. Phys. review letters 114, 230501 (2015).

2. Wagner, K. et al. Magnetic domain walls as reconfigurable spin-wave nanochannels. Nat. nanotechnology 11, $432-436$ (2016). 
3. Jamali, M., Kwon, J. H., Seo, S.-M., Lee, K.-J. \& Yang, H. Spin wave nonreciprocity for logic device applications. Sci. reports 3, 1-5 (2013).

4. Klingler, S. et al. Spin-wave logic devices based on isotropic forward volume magnetostatic waves. Appl. Phys. Lett. 106, 212406 (2015).

5. Buijnsters, F., Ferreiros, Y., Fasolino, A. \& Katsnelson, M. Chirality-dependent transmission of spin waves through domain walls. Phys. review letters 116, 147204 (2016).

6. Kiseki, K., Yakata, S. \& Kimura, T. Efficient excitation and detection of standing spin wave in permalloy film: Demonstration of spin wave resonator. Appl. Phys. Lett. 101, 212404 (2012).

7. Yamamoto, R. et al. External electric field dependence of the structure of the electric double layer at an ionic liquid/au interface. Appl. Phys. Lett. 101, 053122 (2012).

8. Anderson, I. A., Gisby, T. A., McKay, T. G., O’Brien, B. M. \& Calius, E. P. Multi-functional dielectric elastomer artificial muscles for soft and smart machines. J. applied physics 112, 041101 (2012).

9. Prokopov, A. et al. Epitaxial bi-gd-sc iron-garnet films for magnetophotonic applications. J. Alloy. Compd. 671, 403-407 (2016).

10. Kajiwara, Y. et al. Transmission of electrical signals by spin-wave interconversion in a magnetic insulator. Nature 464, 262-266 (2010).

11. Lachance-Quirion, D., Tabuchi, Y., Gloppe, A., Usami, K. \& Nakamura, Y. Hybrid quantum systems based on magnonics. Appl. Phys. Express 12, 070101 (2019).

12. Demidov, V. E., Demokritov, S. O., Rott, K., Krzysteczko, P. \& Reiss, G. Nano-optics with spin waves at microwave frequencies. Appl. Phys. Lett. 92, 232503 (2008).

13. Demidov, V. E. et al. Transformation of propagating spin-wave modes in microscopic waveguides with variable width. Phys. Rev. B 79, 054417 (2009).

14. Schneider, T., Serga, A., Neumann, T., Hillebrands, B. \& Kostylev, M. Phase reciprocity of spin-wave excitation by a microstrip antenna. Phys. Rev. B 77, 214411 (2008).

15. Hansteen, F., Kimel, A., Kirilyuk, A. \& Rasing, T. Nonthermal ultrafast optical control of the magnetization in garnet films. Phys. Rev. B 73, 014421 (2006).

16. Savochkin, I. et al. Generation of spin waves by a train of fs-laser pulses: a novel approach for tuning magnon wavelength. Sci. reports 7, 1-10 (2017).

17. Stupakiewicz, A. et al. Selection rules for all-optical magnetic recording in iron garnet. Nat. communications 10, 1-7 (2019).

18. Stupakiewicz, A., Szerenos, K., Afanasiev, D., Kirilyuk, A. \& Kimel, A. Ultrafast nonthermal photo-magnetic recording in a transparent medium. Nature 542, 71-74 (2017).

19. Lambert, C.-H. et al. All-optical control of ferromagnetic thin films and nanostructures. Science 345, 1337-1340 (2014).

20. Mangin, S. et al. Engineered materials for all-optical helicity-dependent magnetic switching. Nat. materials 13, 286-292 (2014).

21. Atoneche, F. et al. Large ultrafast photoinduced magnetic anisotropy in a cobalt-substituted yttrium iron garnet. Phys. Rev. $B$ 81, 214440 (2010).

22. Jäckl, M. et al. Magnon accumulation by clocked laser excitation as source of long-range spin waves in transparent magnetic films. Phys. Rev. X 7, 021009 (2017).

23. Khokhlov, N. E. et al. Optical excitation of propagating magnetostatic waves in an epitaxial galfenol film by ultrafast magnetic anisotropy change. Phys. Rev. Appl. 12, 044044 (2019).

24. Stanciu, C. et al. Ultrafast spin dynamics across compensation points in ferrimagnetic gdfeco: The role of angular momentum compensation. Phys. Rev. B 73, 220402 (2006).

25. Levy, M. et al. Faraday rotation in iron garnet films beyond elemental substitutions. Optica 6, 642-646 (2019).

26. Kruglyak, V. et al. Imaging small-amplitude magnetization dynamics in a longitudinally magnetized microwire. Phys. Rev. $B$ 77, 172407 (2008).

27. Demidov, V. E., Demokritov, S. O., Rott, K., Krzysteczko, P. \& Reiss, G. Mode interference and periodic self-focusing of spin waves in permalloy microstripes. Phys. Rev. B 77, 064406 (2008). 
28. Chumak, A. V. et al. Spin-wave propagation in a microstructured magnonic crystal. Appl. Phys. Lett. 95, 262508 (2009).

29. Pirro, P. et al. Spin-wave excitation and propagation in microstructured waveguides of yttrium iron garnet/pt bilayers. Appl. Phys. Lett. 104, 012402 (2014).

30. Nikitov, S. A. et al. Magnonics: a new research area in spintronics and spin wave electronics. Physics-Uspekhi 58, 1002 (2015).

31. Sadovnikov, A. et al. Spin wave propagation in a uniformly biased curved magnonic waveguide. Phys. Rev. B 96, 060401 (2017).

32. Sadovnikov, A. et al. Directional multimode coupler for planar magnonics: Side-coupled magnetic stripes. Appl. Phys. Lett. 107, 202405 (2015).

33. Nikitov, S., Tailhades, P. \& Tsai, C. Spin waves in periodic magnetic structures-magnonic crystals. J. Magn. Magn. Mater. 236, 320-330 (2001).

34. Krawczyk, M. \& Grundler, D. Review and prospects of magnonic crystals and devices with reprogrammable band structure. J. Physics: Condens. Matter 26, 123202 (2014).

35. Kirilyuk, A., Kimel, A. V. \& Rasing, T. Ultrafast optical manipulation of magnetic order. Rev. Mod. Phys. 82, 2731 (2010).

36. Chernov, A. et al. Optical excitation of spin waves in epitaxial iron garnet films: Mssw vs bvmsw. Opt. letters 42, 279-282 (2017).

37. Bartels, A., Hudert, F., Janke, C., Dekorsy, T. \& Köhler, K. Femtosecond time-resolved optical pump-probe spectroscopy at kilohertz-scan-rates over nanosecond-time-delays without mechanical delay line. Appl. physics letters 88, 041117 (2006).

\section{Acknowledgements (not compulsory)}

This work was financially supported by a Russian Foundation of Basic Research (project N 20-52-12047) and Deutsche Forschungsgemeinschaft (project No. AK40/11-1). Growth of the iron garnet films was financially supported by the Russian Ministry of Education and Science, Megagrant project N 075-15-2019-1934. Also support by the DFG within the International Collaborative Research Center TRR 160 (project A1 and C2) is appreciated. Anastasiya Khramova acknowledges the support from BASIS Foundation scholarship (18-2-6-202-1). V.I. Belotelov is a member the Interdisciplinary "Scientic and Educational School of Moscow University Photonic and Quantum technologies. Digital medicine.”.

\section{Author contributions statement}

M.K., I.V.S., A.E.K., I.A.A. conducted the experimental part of the study, A.E.K. and V.I.B. prepared the manuscript, A.K.Z., I.A.A., M.B. and V.I.B. conceived the project, M.A.K. was a scientific consultant, A.N.S. and V.N.B. made samples.All authors edited and reviewed the manuscript. 


\section{Supplementary Files}

This is a list of supplementary files associated with this preprint. Click to download.

- Supplementary.docx 